\title{
Response to neoadjuvant chemotherapy in breast cancer in a resource limited environment.
}

\author{
*Ayoade B.A. ${ }^{1}$, Salami B.A. ${ }^{1}$, Oritogun K.S. ${ }^{2}$, Fatungase O.M. ${ }^{3}$, Ebili H.O. ${ }^{4}$, \\ Adekoya A.O.
}

\begin{abstract}
Objective: Assess the outcome of neoadjuvant chemotherapy using Adriamycin and Cyclophosphamide followed by Paclitaxel (AC-P regime) in breast cancer.

Methods: A prospective observational study of newly diagnosed breast cancer patients with palpable breast lumps on neoadjuvant chemotherapy of AC-P regime. Age of the patients, tumour size, stage, estrogen, progestogen and HER2 receptor status were noted. Tumour size measured at presentation, first, third, fifth, sixth and eighth doses to determine response as defined by the UICC method i.e. complete clinical response, partial clinical response, stable disease and progressive disease.
\end{abstract}

Results: Complete clinical response was observed in $40 \%$ of 35 patients studied. Complete clinical response was found in $81.8 \%$ tumours less than $5 \mathrm{~cm}$ in diameter while $20.8 \%$ of tumours greater $5 \mathrm{~cm}$.had complete clinical response. $\left(\mathrm{X}^{2}=11.6, \mathrm{p}=0.001\right)$ Eighty-eight percent complied with treatment schedule. Mastectomy was done in $34.2 \%$, Breast conservation surgery (BCS) in $14.2 \%$, and $17.1 \%$ lost to follow up.

Conclusion: Neoadjuvant chemotherapy using AC-P sequential regime is effective in breast cancer with tolerable side effects and excellent treatment compliance in the study population.

Keywords: Neoadjuvant chemotherapy, breast cancer, clinical response, compliance, limited resource

\author{
*Correspondence Author \\ Ayoade B.A. \\ http://orcid.org/0000-0001-6834-9012 \\ Email: babatunde.ayoade @oouagoiwoye.edu.ng \\ ${ }^{1}$ Department of Surgery, Olabisi Onabanjo University Teaching Hospital Sagamu, Nigeria \\ ${ }^{2}$ Department of Community and Primary Care, Olabisi Onabanjo University Teaching Hospital Sagamu, Nigeria \\ ${ }^{3}$ Department OfAnaesthesia, Olabisi Onabanjo University Teaching Hospital Sagamu, Nigeria \\ ${ }^{4}$ Department of Morbid Anatomy and Histopathology, Olabisi Onabanjo University Teaching Hospital Sagamu, \\ Nigeria
}

\footnotetext{
Research Journal of Health Sciences subscribed to terms and conditions of Open Access publication. Articles are distributed under the terms of Creative Commons Licence (CC BY-NC-ND 4.0). (http://creativecommons.org/licences/by-nc-nd/4.0).

http://dx.doi.org/10.4314/rejhs.v7i2.2
} 


\title{
Réponse à la chimiothérapie néoadjuvante dans le cancer du sein dans un environnement aux ressources limitées.
}

\author{
*Ayoade B.A. ${ }^{\text {, }}$, Salami B.A. ${ }^{1}$, Oritogun K.S. ${ }^{2}$, Fatungase O.M. ${ }^{3}$, Ebili H.O. ${ }^{4}$, \\ Adekoya A.O.
}

\section{Resume}

Objectif: Évaluer les résultats de la chimiothérapie néoadjuvante utilisant de l'Adriamycine et du Cyclophosphamide, suivis du Paclitaxel (régime AC-P) dans le cancer du sein.

Méthodes: Une étude observationnelle prospective de patientes nouvellement diagnostiquées avec un cancer du sein présentant des masses mammaires palpables sous chimiothérapie néoadjuvante du régime AC-P. L'âge des patients, la taille de la tumeur, le stade, l'œstrogène, le progestogène et le statut des récepteurs HER2 ont été notés. Taille de la tumeur mesurée lors de la présentation des première, troisième, cinquième, sixième et huitième doses afin de déterminer la réponse définie par la méthode UICC, à savoir une réponse clinique complète, une réponse clinique partielle, une maladie stable et une maladie évolutive.

Résultats: Une réponse clinique complète a été observée chez $40 \%$ des 35 patients étudiés. Une réponse clinique complète a été trouvée dans $81,8 \%$ des tumeurs de moins de $5 \mathrm{~cm}$ de diamètre, tandis que $20,8 \%$ des tumeurs de plus de $5 \mathrm{~cm}$ avaient une réponse clinique complète. $\left(X^{2}=11,6, p=0,001\right)$ Quatre-vingthuit pour cent ont respecté le programme de traitement. La mastectomie a été pratiquée dans $34,2 \%$ des cas, la chirurgie mammaire conservatrice (BCS) dans $14,2 \%$ des cas et $17,1 \%$ ont été perdus de vue.

Conclusion: La chimiothérapie néoadjuvante à l'aide du traitement séquentiel AC-P est efficace dans le cancer du sein, avec des effets secondaires tolérables et une excellente observance du traitement dans la population étudiée.

Mots-clés: chimiothérapie néoadjuvante, cancer du sein, réponse clinique, compliance, ressources limitées

\author{
*Auteur principal \\ Ayoade B.A. \\ http://orcid.org/0000-0001-6834-9012 \\ Email: babatunde.ayoade@oouagoiwoye.edu.ng
}

\footnotetext{
${ }^{1}$ Department of Surgery, Olabisi Onabanjo University Teaching Hospital Sagamu, Nigeria

${ }^{2}$ Department of Community and Primary Care, Olabisi Onabanjo University Teaching Hospital Sagamu, Nigeria

${ }^{3}$ Department OfAnaesthesia, Olabisi Onabanjo University Teaching Hospital Sagamu, Nigeria

${ }^{4}$ Department of Morbid Anatomy and Histopathology, Olabisi Onabanjo University Teaching Hospital Sagamu, Nigeria
} 


\section{INTRODUCTION}

Breast cancer incidence is rising in the low-income countries (LIC) with attendant increase in mortality and morbidity $(1,2)$. In the high-income countries (HIC) the incidence is higher but the mortality and morbidity is less (2). Many reasons have been adduced for this which includes early diagnosis facilitated by screening programmes, widespread breast cancer awareness, prompt and effective treatment in the HIC. $(1,2,3)$. Whereas in the LIC the disease is characterized by late presentation, coupled with inadequate or non-available facilities for diagnosis and treatment with attendant high mortality and morbidity (3).

Treatment of breast cancer (BC) should be done by a multidisciplinary team consisting of surgeons, radiotherapist, medical oncologist, physiotherapist, pharmacist, radiologist, and oncology nurses(4). It basically consists of locoregional disease control, prevention of systemic recurrence and control of metastases. Locoregional control can be achieved by surgery with or without radiotherapy whereas prevention and control of systemic disease is usually by chemotherapy, use of biologics or hormonal therapy while radiotherapy is useful in control of pain of osseous metastases.

Adjuvant chemotherapy is systemic treatment applied post operatively while neoadjuvant chemotherapy is same treatment applied preoperatively. Neoadjuvant chemotherapy is now commonly used in locally advanced breast cancer.

Neoadjuvant chemotherapy reduces the tumour size and may render locally advanced disease amenable to surgical control $(5,6,7)$, and permit breast conservation surgery in cases hither to considered unsuitable prior to neoadjuvant chemotherapy $(5,6,7)$. Another advantage of neoadjuvant chemotherapy is the opportunity to assess tumour response to the drugs being applied $(5,6,7)$. Despite these advantages there is no difference in overall survival in patents who had adjuvant therapy and those who had neoadjuvant chemotherapy (7).

There are many chemotherapy regimens for breast cancer therapy, from single agent to combination therapy. Choice of the agents and regime used depends on the aims of therapy, the stage of the disease, the hormone(oestrogen and progestogen)and HER 2 receptors status, the physiological status of the patient, availability and affordability(8). Many of the agents and regimes adopted for use in the developing world were selected from clinical trials conducted in the developed world.

In the developed world the advent of newer drugs and the practice of personalised medicine has reduced morbidity and mortality of breast cancer. The high cost, non-availability of these drugs and practices has been a major impediment to their use in the developing countries (9).

Most of our patients present with locally advanced breast cancer who often require neoadjuvant chemotherapy using regimes whose effectiveness were based on clinical trials among Caucasians.

The aim of our study was to assess the outcome of neoadjuvant chemotherapy in the management of breast cancer in our practice.

\section{Objectives:}

1.Assessment of clinical response to chemotherapy regime Adriamycin, Cyclophosphamide, followed by Paclitaxel (AC-P), in neoadjuvant therapy for breast cancer in our clinical setting.

2. To identify common side effects.

3. Factors affecting drug compliance.

There is paucity of data on the outcome of neoadjuvant chemotherapy using these agents in patients with breast cancer. We are not aware of any previous study assessing neoadjuvant chemotherapy in our locality. The regimes were chosen on account of effectiveness among Caucasian population, low cost and easy availability in the country compared to other regimes.

\section{MATERIALS AND METHODS}

This is a prospective observational study of patients on neoadjuvant chemotherapy for newly diagnosed breast cancer patients with palpable lumps attending Olabisi Onabanjo University Teaching Hospital Sagamu, Breast, Endocrine and Surgical Oncology unit from $1^{\text {st }}$ July 2017 to $31^{\text {st }}$ December 2018.

The study was approved by the Hospital Ethics Review Committee and patients written consent were obtained,

Inclusion criteria: All patients with histologically confirmed breast cancer and palpable mass on neoadjuvant chemotherapy who consented.

Exclusion criteria: Patients who declined consent, those with metastatic disease, and those who were judged clinical unfit were excluded. 
The demographic characteristics of the patients were noted, symptoms and duration at presentation, findings on clinical examination with particular attention to the breasts stating lump size measured at the widest diameter and its perpendicular diameter using calipers, site, state of axillary nodes, clinical staging by TNM/AJCC method(10), histological type and grading, ER,PR, and HER II receptors status. Routine investigations to assess fitness for chemotherapy like FBC, E\&U, ECG , chest x-ray were done. These are all routine investigations adopted in our practice.

The regime of four courses of I.V. Adriamycin $60 \mathrm{mg} / \mathrm{m} 2$ and I. V Cyclophosphamide $600 \mathrm{mg} / \mathrm{m} 2$ at three weekly interval was followed by four courses of I.V paclitaxel $175 \mathrm{mg} / \mathrm{m} 2$ at three weekly interval with the standard precautions of premedication with dexamethasone and metoclopramide (11). The routine investigations of FBC, E\&U and LFT was done before each cycle.

Response to the therapy was assessed by measuring the size of the lump at its widest diameter and its perpendicular diameter after the first dose, the third dose, sixth (at the commencement of the next dose) and last dose by the principal investigator or the first coinvestigator who have been doing this as part of our routine practice. The response to therapy was assessed using the UICC method consisting of the four categories: complete clinical response, partial clinical response, stable disease and progressive disease.(12) Patients who show progressive disease on this regime who were not suitable for surgical treatment were referred to the Radiotherapist/Oncologist. Side effects observed were noted. The above is our normal protocol for administration of Neoadjuvant therapy.

Compliance with the regime and reasons for non-compliance were noted which were assessed at each follow up visit.

Data collection: Data were collected on specially designed forms and subsequently analyzed using SPSS software version 20.

Data analysis: SPSS Software was used to analyze outcome measures such as response to therapy in four UICC categories which were further classified as good clinical response which consists of complete clinical response or poor clinical response which consists of partial clinical response, stable disease and progressive disease, frequency of side effects, compliance/adherence and reasons for non-adherence using percentages, Chi square to test the significance of the association of the paired categorical variables and level of significance set at $p<.05$

\section{RESULTS}

Thirty-five female patients were seen during the study period. Their age range was 3382 years and mean age of 48 with Standard deviation (SD)of $11,37.1 \%$ were in the 41 to 50 years age bracket. Forty percent had education up to secondary school level, $65.7 \%$ were premenopausal, $62.9 \%$ were traders The rest of the demographic features are as in Table 1. The presenting symptom was lump in the breast in all the patients but one had a nipple discharge and the lump was ulcerated in $17.6 \%$. The range of symptoms duration was 1-24 months, (mean 7.5 months, SD 6.5months), lump size range was 2$24 \mathrm{~cm}$ (mean $11.3 \mathrm{~cm}$, SD $7.5 \mathrm{~cm}$ ). Twenty-nine patients $(82.9 \%$ ) had AJCC Stage III disease, the rest as in Table 2. All the patients had invasive ductal carcinoma. All the patients had between six to eight courses of chemotherapy of the AC-P regime.

The overall clinical response rate to neoadjuvant chemotherapy in this study was $80 \%$, consisting of complete clinical response of $40 \%$ and partial clinical response $40 \%$. Stable disease and progressive disease were observed in $8.6 \%$ and $11.4 \%$ of the patients respectively. Complete clinical response observed in $40 \%$ was classified as Good clinical response and Partial response observed in $40 \%$, Stable disease in $8.6 \%$ and Progressive disease in $11.4 \%$ were classified as Poor clinical response. The comparison of complete clinical response and clinical parameters is shown in Table 3. The clinical response in the AJCC stage and immunohistochemistry is as shown in Table 3.

The most common side effects of the drugs were, nausea, alopecia, hyperpigmentation of the hands and feet and the rest as shown in Table 4.Thirty-one patients $(88.6 \%)$ complied with the treatment protocol while four did not. Reason for non-compliance was cost in 3 patients $(8.6 \%)$ and side effects in one patient. Mastectomy was done in 12patients (34.2\%), Breast conservation surgery in 5patients $(14.2 \%)$, 7 patients $(20 \%)$ are awaiting surgery as at the time of this report, 5patients (14.2\%) were referred to Radiation oncologist, 6patients (17.1\%) absconded from follow up of which 3 had complete clinical response. 


\section{DISCUSSION}

The majority of patients in this study were premenopausal women with mean age of 48 years and over a third of them in the $41-50$ years age bracket. This is consistent with observed age incidence in breast cancer in sub Saharan Africa unlike in the western world where older women are more affected(13). The cohort also consisted of mostly patients with locally advanced disease, a group in which neoadjuvant chemotherapy is strongly indicated. Late presentation evidenced by long duration of symptoms and presence of large palpable lumps as seen in this study is the usual occurrence(3), moreover this observation is expected since one of the inclusion criteria is the presence of palpable lumps.

The overall clinical response observed in this study compares favourably with findings of Awad Ali in Sudan who found a clinical response rate of $83 \%$ in a prospective study of 98 patients on chemotherapy(14), and other workers (15). This demonstrates the effectiveness of these agents in reducing the gross tumour size in the study population. This is at variance with the findings of Arowolo et al in a retrospective review of 62 breast cancer cases on neoadjuvant chemotherapy seen over a 24 year period in which clinical response was observed in $51.7 \%$ of the patients(16).

Complete clinical response was observed in $40 \%$ of the patients, this is comparable to values quoted by other workers for Adriamycin and cyclophosphamide combination chemotherapy $(17,18)$ though our regime included four 3 weekly course of paclitaxel, a taxane which has been found to improve clinical response.(19). Clinical response determined by palpation or by radiological investigations like ultrasound, mammography or Breast MRI may not accurately assess presence or absence of residual tumour after neoadjuvant chemotherapy since residual fibrosis or tissue oedema may occur and also patchy or scattered tumour residue may be present.(17,18,20). A better measure of response to neoadjuvant chemotherapy is complete pathological response which is determined by histopathological examination of postoperative specimen for residual tumour after neoadjuvant therapy. Complete pathological response after neoadjuvant chemotherapy has been associated with prolonged disease free period and better overall survival.(21) One of the advantages of neoadjuvant therapy is the in vivo demonstration of efficacy of the drugs and appropriate measures can be instituted when there is lack of efficacy to avoid futile treatment(22). In this study, $20 \%$ of the patients showed either stable or progressive disease interpreted as no response and those not suitable for surgical treatment were promptly referred to Radiotherapist/Clinical oncologist.

Several factors have been studied to predict clinical response to neoadjuvant chemotherapy such as age, menstrual status, size of tumour, stage of disease, tumour grade, ER, PR, HERII, Ki67, apoptosis related gene $p 53, b c l$ 2 and $B A X$ but so far the data available is limited(23). In our study $70 \%$ of the patients aged 40years and below had good clinical response compared to $28 \%$ in patients aged above forty years, also $52.2 \%$ of patient who were premenopausal had good clinical response compared with $16.6 \%$ in post menopausal women, ( both of this observation were statistically significant).Good clinical response by younger patients as seen in our study has been reported by other researchers $(24,25)$. Good clinical response was observed in $81.8 \%$ of patients with tumours less than $5 \mathrm{~cm}$ in diameter, compared to $20.8 \%$ with good clinical response in those with tumours greater than $5 \mathrm{~cm}$ in diameter, this was statistically significant $\mathrm{Chi}$ squared 11.6, $\mathrm{p}=.001$. Bonadonna et al observed similar findings in which, tumour size had an inverse relationship to complete clinical response.(26). The reason is likely related to the fact that smaller tumours usually have larger growth fraction consisting of rapidly dividing cells which are principally affected by chemotherapeutic agents. This is also reflected in the observation that all the Stage 2 tumours had good clinical response compared with $27.6 \%$ (8 0f 29) cases with stage 3 tumours.

Triple negative breast cancer (TNBC), hormone receptor negative and HER II positive breast tumours show good response to neoadjuvant chemotherapy (27), but in our study only $25 \%$ (3of 12 ) cases of TNBC had good response compared with Non-TBNC tumours $46 \%$ (6 of 13) had good clinical response. This observation may be due to the small number of cases studied which was reduced further by unavailability of immunohistochemistry result for $28.6 \%$ (10 of 35$)$ of the cases.

The common side effects observed in this study included gastrointestinal symptoms like vomiting and anorexia, hyperpigmentation of the hands and feet, and alopecia which was almost universal, similar to observation of CleggLamptey et-al in Ghana and Fisher et.al $(28,29)$. These side effects occurred despite administration of metoclopramide and 
dexamethasone as premedication in all the patients, however none of the patient had severe reaction necessitating discontinuation. Haematological side effects such as leucopenia, anaemia and thromobocytopeania were also observed in the study but with less frequency than gastrointestinal symptoms and they were all successfully managed with dose deferment for one to two weeks, while leucopenia and anaemia were treated using Filgrastim and blood transfusion respectively There was no recourse to dose reduction.

Compliance with treatment schedule was observed in 31 patients (88.\%), the three patients who had interruptions due to inability to pay for their drugs and one who missed appointment on account of side effects returned later to continue the treatment. This high compliance rate is different from the report of others like Egwuonwu et al (30) and Cleggy-Lamptey et al.(28). The possible explanation for this may be the current affordability, availability of these drugs and intensive pretreatment counselling of the patients.

Disease progression has been said to be one of the drawbacks of neoadjuvant therapy in which unresponsive tumour may progress during therapy and become unsuitable for surgical therapy. Early assessment of response and prompt change of treatment strategy will obviate this. In this study $14.2 \%$ who had progressive disease were so referred.

Absconding from further treatment is common in our practice, and various reasons have been adduced for this including ignorance, fear of surgery in this case mastectomy, spiritual, sociocultural factors and economic reasons $(28,30)$. In our study five patients absconded, three of which had complete clinical response and felt there was no reason to have further treatment since the lesions were no longer palpable despite repeated counselling on telephone.

Study Limitations: The small number of the study population which makes the study lack sufficient power. This is a report without long time outcome data such as disease-free interval and overall survival which can only be available after long time follow up.

\section{CONCLUSION}

Neoadjuvant chemotherapy using AC-P regime is effective in breast cancer treatment with tolerable side effects and excellent treatment compliance in the study population.

Conflict of interest: The authors declare no conflicts of interest.

\section{REFERENCES}

1. Anderson BOJ, Yip $\mathrm{CH}$, Smith RA, et al Guidelines implementation for breast health care in low-income and middle-income countries. Overview of Breast Health Global Initiative Global Summit 2007. Cancer 2008. 113:2221-43

2. Adebamowo CA, Ajayi OO, Breast Cancer in Nigeria. West Afr J Med 2000;19:179-91

3. Ayoade BA, Agboola AJ, Olatunji AA, et al. Clinical characteristics and survival outcome of Breast Cancer in Southwest Nigerian women. J. Afr. Cancer 2014; 6:79-84. DOI 10.1007/s12558014-0311-8

4. Kesson EM, Allardice GM, George WD, et al. Effects of multidisciplinary team working on breast cancer survival: retrospective, comparative, interventional cohort study of 13722 women. BMJ 2012;344:e2718

5. Gralow JR, Burstein HJ, Wood W, et al .Preoperative therapy in invasive breast cancer: pathologic assessment and systemic issues. J Clin Oncol 2008; 26:814

6. Kaufmann M, Hortobagyi GN, Goldhirsch A, et al. Recommendations from an international expert panel on the use of neoadjuvant (primary) systemic treatment of operable breast cancer: an update. J.Clin Oncol 2006;24:1940

7. Schwartz GF, Hortobagyi GN. Proceedings of consensus conference on neoadjuvant chemotherapy in carcinoma of the breast, April 26-28 2003, Philadephia, Pennsylvania. Cancer 2004; 100:2512

8. [Guideline] NCCN Clinical Practice Guidelines in Oncology. Breast Cancer. Version1.2017. National Comprehensive Cancer Network. A v a i 1 a b 1 e a t http:/www.ncn.org/professional/physician_gls/p df/breast.pdf. March 10; Accessed on April 14.2017

9. World Health Organization. National cancer control programmes. Geneva: World Health Organization; 2002.

10. Sobin LH, Wittekind CH, editors. UICC: TNM classification of malignant tumours. $6^{\text {th }}$ ed.New York: Wiley-Liss; 2002

11. Sparano JA, Wang M, Martino S, et al. Weekly Paclitaxel in the adjuvant treatment of Breast cancer. N Eng J Med 2008: 358: 1663

12. Hayward JL, Carbone PP, Heuson JC, Kumaoka S, Segaloff A, Rubens RD,. Assesment of response to therapy in advanced breast cancer: a project of the Programme on Clinical Oncology of the International Union Against Cancer, Geneva Switzerland. Cancer 1977; 39: 1289-94

13. Oluwole SF, Fadiran OA, Odesanmi WO. Diseases of the breast in Nigeria. Br J Surg 1987; 74: $582-5$

14. Awad Ali. M .A. Evaluation of clinical and Pathological response after two cycles of 
neoadjuvant chemotherapy on Sudanese patients with locally advanced breast cancer. Ethiop J Health Sci. 2014 ;24(1) 16-20

15. Wolmark N, Wang J, Mamounas E, Bryant J, Fisher B. Preoperative chemotherapy in patients with operable breast cancer: nine -year result from National adjuvant breast and bowel project B-18. Journal of the National Cancer Institute Monograph 2001;30:96-102

16. Arowolo OA, Akinkuolie AA, Lawal OO, Alatise OI, Salako AA, Adisa AO. The impact of Neoadjuvant Chemotherapy on Patients with Locally advanced Breast Cancer in a Nigerian Semi urban Teaching Hospital: A single center Descriptive study. World J Surg. 2010; 34: 17711778

17. Fisher ER, Wang J, Bryant J, Fisher B, Mamounas E and Wolmark N. Pathology of preoperative chemotherapy: Findings from National Surgical Adjuvant Breast and Bowel (NASBP) protocol B18. Cancer , 2002;95:681-695

18. Honkoop AH, Lukx-de Bakker SA, Hoekman K eet al. Prolonged neoadjuvant chemotherapy with GM-CSF in locally advanced breast cancer. Oncologist, 1999; 4: 106-111

19. Bear HD, Anderson S, Smith RE, Geyer CE Jr, Mamounas EP, Fisher B, Brown Am, et al Sequential preoperative or postoperative docetaxel added to preoperative doxorubicin plus cyclophosphamide for operable breast cancer. National Surgical Adjuvant Breast and Bowel Project Protocol B-27. J Clin Oncol 2006;24(13): 2019-2027

20. Chollet P, Charrier S, Brian E et al. Clinical and pathological response to primary neoadjuvant chemotherapy in operable breast cancer. Eur J Cancer, 1997; 33: 862-866

21. Kaufman M, Von Minckwitz G, Bear HD, et al. Recommendations from an international expert panel on the use of neoadjuvant (primary) systemic treatment of operable breast cancer: new perspectives 2006. Ann Oncol 2007; 18(12): 1927-1934

22. Untch M, von Minckwitz. Neoadjuvant Chemotherapy: Early response as a Guide for further treatment: Clinical, Radiological and biological. J Natl Cancer InstMonogr 2011;43:138-141

23. Tewari M, Krishnamurthy A, Shukla HS. Predictive markers of response to neoadjuvant chemotherapy in Breast cancer. Surgical Oncology 2008; 17:301-311

24. HuoberJ, von Minckwitz G, Denkert C, et al: Effect of neoadjuvant anthracycline-taxane based chemotherapy in different biological breast cancer phenotypes: overall results from the GeparTrio study. Breast Cancer Res Treat 2010; 124: $133-140$

25. Loibl S, Jackish C, Gade S, et al Neoadjuvant chemotherapy in the very young 35 years of age or younger. Cancer Res 2012;72 (suppl24):S3-1

26. Bonnadonna G, Valgussa P, Zucali R, SalvadoriB. Primary chemotherapy in surgically resectable breast cancer. CA; A cancer Journal for clinicians ; 45: $227-43$

27. Esserman LJ, Berry DA, Cheang MC, et al. Chemotherapy response and recurrence- free survival in neoadjuvant breast cancer depends on biomarker profiles: results from I-SPY1 Trial (CALGB 150007/150012). Breast Cancer Res Treat 2012;132: 1049-1062

28. Clegg-Lamptey J, Hodasi W. A study of breast cancer in Korle Bu Teaching Hospital: Assessing the impact of health education. Ghana Med J 2007;41: 72-7

29. Fisher B, Brown AM, Dimitrov NV, Poisson R, Redmond C, Margolese RG, et.al, Two months of doxorubicin-cyclophosphamide with and without interval reinduction therapy compared with 6months of cyclophosphamide, methotrexate and fluorouracil in positive-node breast cancer patients with tamoxifen- nonresponsive tumours: Results from the National Surgical Adjuvant Breast and Bowel Project B-15. J Clin Oncol 1990;8:1483-96

30. Egwuonwu OA, Anyanwu SNC, Nwofor AME. Default from Neoadjuvant chemotherapy in premenopausal female breast cancer patients: What is to blame? Niger Jour Clin Pr. 2012;15:265-269 
Table 1: Demographic features

\begin{tabular}{lll}
\hline & Frequency & Percentage (\%) \\
\hline Age groups (years) & 10 & 28.6 \\
$<40$ & 13 & 37.1 \\
$41-50$ & 9 & 25.7 \\
$51-60$ & 1 & 2.9 \\
$61-70$ & 2 & 5.7 \\
$>70$ & 35 & 100 \\
Total & & \\
Menstrual status & 23 & 65.7 \\
Premenopausal & 12 & 34.3 \\
Postmenopausal & 35 & 100 \\
Total & & \\
Educational Level & 4 & 11.4 \\
No formal education & 5 & 14.3 \\
Primary & 14 & 40.0 \\
Secondary & 12 & 34.3 \\
Tertiary & 35 & 100 \\
Total & & \\
Occupation & 22 & 62.9 \\
Trading & 4 & 11.4 \\
Civil servant & 4 & 11.4 \\
Teaching & 2 & 5.7 \\
Artisan & 2 & 5.7 \\
Unemployed & 1 & 2.9 \\
Nursing & 35 & 100 \\
Total & & \\
\hline
\end{tabular}

Table 2. AJCC stage and Immunohistochemistry

\begin{tabular}{ccl}
\hline & Frequency & Percentage (\%) \\
\hline AJCC Stage & 2 & \\
I & 4 & 5.7 \\
II & 29 & 11.4 \\
III & 35 & 100.0 \\
Total & & \\
Immunohistochemistry & 12 & 48.0 \\
TNBC & 3 & 12.0 \\
Er+, Pr+, HER+ & 2 & 8.0 \\
Er+,Pr+, HER- & 7 & 28.0 \\
Er-, Pr-, HER+ & 1 & 4.0 \\
Er+, Pr-, Her- & 25 & 100.0 \\
Total & &
\end{tabular}


Table 3. Clinical response and clinical parameters such as age, menstrual status, size, Stage and Immunohistochemistry

\begin{tabular}{|c|c|c|c|c|}
\hline & $\begin{array}{l}\text { Good Clinical } \\
\text { response }(\%)\end{array}$ & $\begin{array}{l}{ }^{\text {aPoor clinical }} \\
\text { response }(\%)\end{array}$ & $\begin{array}{l}\text { Total } \\
(\%)\end{array}$ & $\mathrm{X}^{2}$ \\
\hline \multicolumn{5}{|l|}{ Age } \\
\hline$<40$ years & $7(70.0)$ & $3(30)$ & $10(100)$ & 5.25 \\
\hline$>40$ years & $7(28.0)$ & $18(72.0)$ & $25(100)$ & $\mathrm{P}=.02$ \\
\hline Total & $14(40.0)$ & $21(60.0)$ & $35(100)$ & \\
\hline \multicolumn{5}{|l|}{ Menstrual status } \\
\hline Premenopausal & $12(52.2)$ & $11(47.8)$ & $23(100)$ & 4.14 \\
\hline Postmenopausal & $2(16.6)$ & $10(83.3)$ & $12(100)$ & $\mathrm{P}=.04$ \\
\hline Total & $14(40.0)$ & $21(60.0)$ & $35(100)$ & \\
\hline \multicolumn{5}{|l|}{ Size } \\
\hline$<5 \mathrm{~cm}$ & $9(81.8)$ & $2(18.2)$ & $11(100)$ & 11.6 \\
\hline$>5 \mathrm{~cm}$ & $5(20.8)$ & $19(79.2)$ & $24(100)$ & $\mathrm{P}=.001$ \\
\hline Total & $14(40.0)$ & $21(60.0)$ & $35(100)$ & \\
\hline \multicolumn{5}{|l|}{ AJCC Stage } \\
\hline 2 & $6(100)$ & $0(0.0)$ & $6(100)$ & \\
\hline 3 & $8(27.6)$ & $21(72.4)$ & $29(100)$ & \\
\hline Total & $14(40.0)$ & $21(60.0)$ & $35(100)$ & \\
\hline \multicolumn{5}{|l|}{ Immunohistochemistry } \\
\hline $\mathrm{TNBC}$ & $3(25.0)$ & $9(75.0)$ & $12(100)$ & 1.6 \\
\hline${ }^{b}$ Non-TNBC & $6(46.0)$ & $7(54.0)$ & $13(100)$ & Fisher's \\
\hline Total & $9(36.0)$ & $16(64.0)$ & $25(100)$ & $\begin{array}{l}\text { Exact test } \\
\mathrm{P}=.400\end{array}$ \\
\hline
\end{tabular}

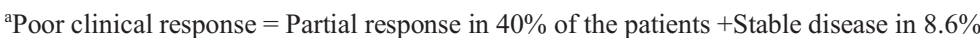
of the patients + Progressive disease in $11.4 \%$ of the patients.

${ }^{b}$ Non- $\mathrm{TNBC}=$ Tumours with any of these ER,$+ \mathrm{PR}+, \mathrm{HER}+$

Table 4. Frequency of Side effects

\begin{tabular}{llc}
\hline Side effect & \multicolumn{2}{l}{ Number $(\%)$} \\
\hline GIT symptoms, Nausea, vomiting, anorexia & 29 & $(82.9)$ \\
Alopecia & 33 & $(94.3)$ \\
Hand and foot hyperpigmentation & 33 & $(94.3)$ \\
Neutropenia & 18 & $(51.4)$ \\
Thrombocytopenia & 9 & $(25.7)$ \\
Anaemia & 18 & $(51.4)$ \\
Cardiac & 3 & $(8.6)$ \\
Neuropathy & 4 & $(11.4)$ \\
\hline
\end{tabular}

